\title{
TRANSFORMATION OF AlGaAs/GaAs INTERFACE UNDER HYDROSTATIC PRESSURE
}

\author{
J. Bąk-Misiuk, J. Domagala, J. Trela \\ Institute of Physics, Polish Academy of Sciences \\ Al. Lotników 32/46, 02-668 Warszawa, Poland \\ M. LESZCZYŃSKI
}

High Pressure Research Center, Polish Academy of Sciences

Sokolowska 29/37, 01-142 Warszawa, Poland

\section{A. Misiuk}

Institute of Electron Technology, Al. Lotników 32/46, 02-668 Warszawa, Poland

\section{J. Härtwig and E. Prieur}

European Synchrotron Radiation Facility, BP220, 38043 Grenoble Cedex, France

AlGaAs layers grown by molecular beam epitaxy on GaAs substrates were investigated before and after high hydrostatic pressure $(1.2 \mathrm{GPa})$ at high temperature $(770 \mathrm{~K})$ treatment (HP-HT treatment). An influence of HP-HT treatment on the properties of the AlGaAs/GaAs system was studied by lattice parameter measurements using the high resolution diffractometer and by X-ray topography. Observed changes in the lattice parameter of the AlGaAs layers after HP-HT treatment are related to the strain relaxation and explained by the creation of misfit dislocations and other extended defects which are visible on the topographs.

PACS numbers: $81.40 .-z, 65.70 .+y$

\section{Introduction}

It has been established that single crystals of $\mathrm{Si}, \mathrm{GaAs}$ and $\mathrm{GaP}$ [1-3] containing inhomogeneities in compressibility and thermal expansion values (different from those of the matrix) can change their defect structure under high hydrostatic pressure (HP) at high temperature (HT). This effect is due to the enhanced strain at the precipitate/matrix boundary caused by HP. For the stress value above the critical one additional defects can be created. The influence of HP on the relaxation behaviour of strained layers has been investigated at HT for the 
$\mathrm{Si}_{1-x} \mathrm{Ge}_{x} / \mathrm{Si}$ system [4]. The influence of HP-HT treatment on the lattice parameters of semiconductors can be important for the determination of lattice mismatch in superlattices or heterostructures.

$\mathrm{AlGaAs}$ is the most frequently applied ternary semiconductor. Its direct energy gap and good lattice match with GaAs substrate have made it an almost perfect material for heterostructure and superlattice technologies for electronic and optoelectronic applications.

\section{Experimental}

$3 \mu \mathrm{m}$ thick $\mathrm{Al}_{1-x} \mathrm{Ga}_{x} \mathrm{As}$ layers $(x=0.27)$ grown by molecular beam epitaxy (MBE) at temperature about $1000 \mathrm{~K}$ on the (001) oriented LEC-grown (liquid encapsulated Czochralski) GaAs substrates were subjected to HP (1.2 GPa) at HT (770 K) during $1 \mathrm{~h}$. X-ray measurements were carried out for the samples before and after HP-HT treatment using high resolution diffractometry (MRD-Philips) and synchrotron X-ray diffraction topography at D5 Optics beamline at the European Synchrotron Radiation Facility (ESRF).

The lattice parameters perpendicular and parallel to the sample interfaces were measured by the Bond method in the temperature range of $293-673 \mathrm{~K}$. In order to further investigate the structural properties of the layers, reciprocal space maps were made using symmetrical 004 and asymmetrical 444, 115 and 335 reflections. If the lattice planes of the epilayer are not tilted with respect to those of the substrate, the reciprocal space maps of the asymmetrical reflections give direct information about the strain state of the layer [5].

\section{Results}

The lattice parameters parallel to the interface, $a_{\|}$, for all investigated layers were found to be equal to the lattice parameters of the substrates over the whole used temperature range. This means that the layers are strained. The same result was deduced from the reciprocal space maps of the symmetrical 004 (Fig. 1a)
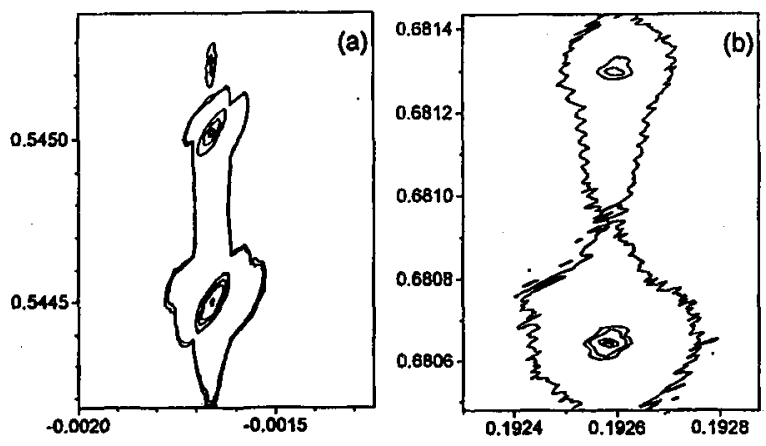

Fig. 1. Reciprocal space maps of HP-HT treated AlGaAs/GaAs sample for the symmetrical 004 (a) and asymmetrical 115 (b) reflections. The axes are marked in units $\lambda / 2 d$. 


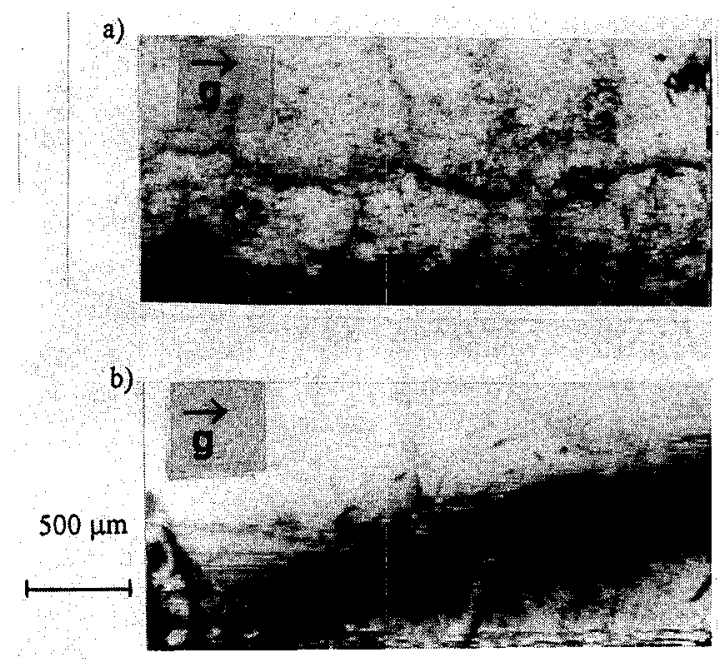

Fig. 2. Transmission topographs of as-grown (a) and HP-HT treated (b) samples taken for diffraction vector $g=[022]$ and X-ray wavelength $\lambda=0.39 \AA$. Scale and $g$ are marked in the figure.

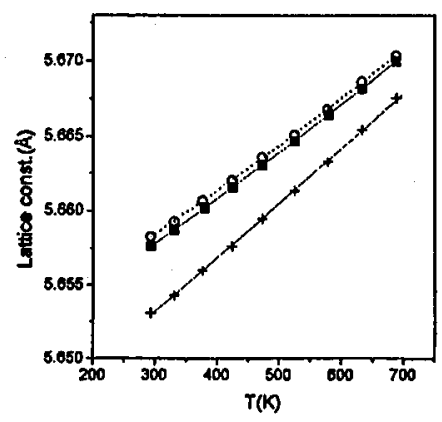

Fig. 3. Temperature dependence of lattice constant, $a_{\perp}$, for $\mathrm{Al}_{0.27} \mathrm{Ga}_{0.73} \mathrm{As} / \mathrm{GaAs}$ system: (+) substrate, ( $)$ as-grown sample, (0) HP-HT treated sample.

and the asymmetrical 115 (Fig. 1b) reflections. Because no tilt was found when mapped the reciprocal space of the symmetrical 004 reflection, the asymmetrical 115 reciprocal space map gives direct information about the strain state of the epilayer.

The result that the layer is strained is rather unexpected because for the HP-HT treated sample the misfit dislocation lines (parallel to the [011] direction) were observed in the X-ray topographs (Fig. 2b). Figure 2b shows also many other defects created during or after HP-HT treatment. Topographs of as-grown samples show no misfit dislocations (Fig. 2a). Only a cellular structure of dislocations, which is typical of LEC-grown GaAs substrates, is visible in the topographs. Lattice parameter perpendicular to the interface, $a_{\perp}$, is higher for HP-HT than that for the as-grown samples in the whole temperature range (Fig. 3). 


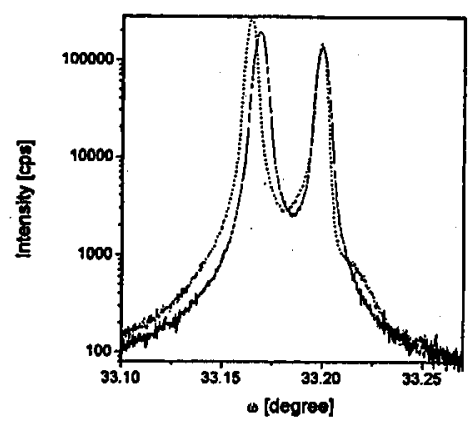

Fig. 4. Rocking curves of $004 \mathrm{Cu} K_{\alpha_{1}}$ reflection from $\mathrm{Al}_{0.27} \mathrm{Ga}_{0.73}$ As layers on $\mathrm{GaAs}$ substrate. Solid and dashed lines correspond to as-grown and HP-HT treated samples, respectively.

This difference $\left(5.4 \times 10^{-4} \AA\right)$ is practically independent on the temperature, and therefore the thermal expansion coefficients $\left(\alpha_{\perp}\right)$ for as-grown and HP-HT treated samples are constant and equal to $(4.21 \pm 0.02) \times 10^{-6} \mathrm{~K}^{-1}$. Rocking curves of as-grown and HP-HT treated samples are shown in Fig. 4. Small broadening (about 2 arcsec) of rocking curve full width of half maximum (FWHM) for the substrate and the layer after HP-HT treatment was found for symmetrical and asymmetrical reflections. Also the additional oscillations are visible but only on the large angle side. One of these oscillations is visible also on the 004 reciprocal space map as an additional point (see Fig. 1a).

\section{Discussion}

The investigated as-grown AlGaAs layers were under compressive strain. It results from the different lattice parameters for the AlGaAs layer and $\mathrm{GaAs}$ substrate. During the layer deposition as well as after cooling the sample, the layer and the substrate were matched (lack of misfit dislocations at the interface) but, because of the lattice parameters difference, the layer was squeezed. After HP-HT processing the layer remained in the compressed state but from the observation that $a_{\perp(0)}<a_{\perp(\mathrm{HP}-\mathrm{HT})}$ and $a_{\|(0)}=a_{\|(\mathrm{HP}-\mathrm{HT})}$ it follows that the unit cell volume and strain after HP-HT processing are higher than those before. On the other hand, there are misfit dislocations on the X-ray topographs of the processed samples. This means that at the HP-HT conditions the strain at the interface was high enough to cause partial or full relaxation of the layer. A possible explanation for the creation of misfit dislocations at temperature $T<T_{\text {layer growth }}$ is the following: it is assumed that at the HP-HT conditions the layer is fully relaxed through the creation of the misfit dislocations and that strain, $\varepsilon$, at such conditions would be equal to 0 . During the cooling, the layer stays "matched" to the substrate but the created dislocations remain. At HP-HT conditions the strains $\left(\varepsilon_{\mathrm{HP}-\mathrm{HT}}\right)$ at the interface between thin layer and substrate can be described [6] by the formula:

$$
\varepsilon_{\mathrm{HP}-\mathrm{HT}}=\Delta T\left(\alpha_{\mathrm{L}}-\alpha_{\mathrm{S}}\right)+(P / 3)\left(1 / B_{\mathrm{S}}-1 / B_{\mathrm{L}}\right),
$$

where $\alpha_{\mathrm{S}}$ and $\alpha_{\mathrm{L}}$ are thermal expansion coefficients for the substrate and the layer, and the $B_{\mathrm{S}}, B_{\mathrm{L}}$ are the bulk moduli for the substrate and the layer, respectively. 
According to the model of the process described by the above formula, at the applied HP-HT conditions the strain should be lower than that during the growth of the layer (because $B_{\mathrm{S}}>B_{\mathrm{L}}$ and $\alpha_{\mathrm{S}}>\alpha_{\mathrm{L}}$ ).

Therefore the creation of dislocations of temperature below that of the layer growth can be explained as follows: at HP-HT conditions the critical strain for some HP value dependence on temperature is shifted to lower strain values. It means that when $T$ rises, the critical strain for the dislocation generation is reached below the layer growth temperature and dislocations are created.

As it is seen in the topographs, HP-HT processed sample contains a high concentration of defects, probably inclusions or precipitates. It is known that HP applied to such a system can result in creation of additional defects leading to the rise of the lattice parameter $[1,3]$. It seems that just this phenomenon (creation of additional defects) is responsible for the higher $a_{\perp}$ value and unit cell volume in the AlGaAs layer and so for the higher compressive strain in the HP-HT processed AlGaAs/GaAs system.

A possible explanation for the observed additional oscillations in the 004 rocking curve of HP-HT treated samples is gradient of lattice constant induced, for example, the enhanced diffusion of $\mathrm{Al}$ atoms from the surface to the interface leading to the non-uniform $\mathrm{Al}$ concentration in the layer. Similar observation has been reported in [4].

\section{Summary}

It was shown that HP-HT treatment of $\mathrm{AlGaAs} / \mathrm{GaAs}$ heterostructures causes the creation of misfit dislocations and increases the relaxed lattice parameter of the layer.

\section{Acknowledgment}

This work was supported by grants of the Committee for Scientific Research (numbers: 101/P3/93/05, 288/P4/94/06, 8 T11B 048 09/95) and by Polish-French Cooperation Joint Project no. 5197. The Philips MRD diffractometer was founded by the Foundation for Polish Science (program SEZAM 94).

\section{References}

[1] A. Misiuk, J. Wolf, L. Datsenko, J. Adamczewska, J: Bąk-Misiuk, Nukleonika 39, 281 (1994).

[2] J. Bakk-Misiuk, A. Misiuk, J. Jabłoński, A. Moroz, A. Morawski, Acta Phys. Pol. A 73, 505 (1988).

[3] J. Bąk-Misiuk, J. Domagała, B. Kozankiewicz, A. Misiuk, J. Adamczewska, M. Skibska, Acta Phys. Pol. A 83, 87 (1993).

[4] P. Zaumseil, G.G. Fischer, Ch. Quick, A. Misiuk, Solid State Phenomena 47-48, 517 (1995).

[5] H. Heinke, A. Waag, M.O. Möller, M.M. Requet, G. Landwehr, J. Cryst. Growth 135, 53 (1994).

[6] J. Jung, Philos. Mag. A 50, 257 (1984). 